\title{
Rethinking the Concept of Just Noticeable Difference in Online Marketing
}

\author{
Viktor Vojtko ${ }^{1}$ \\ ${ }^{1}$ Department of Trade and Tourism, Faculty of Economics, \\ University of South Bohemia in Ceske Budejovice \\ Studentska 13, 37005 Ceske Budejovice \\ vojtko@ef.jcu.cz
}

\begin{abstract}
The main goal of this study is to answer a question whether the just noticeable difference (JND) related marketing practices could survive in the world with social media and as a part of online marketing. Although the findings are limited, they suggest that using of such practices might be much riskier than it used to be before and marketers should be aware of that and consider their using more thoroughly. It also shows that usage of the agent based modelling (ABM) can be helpful in dealing with problems like this one and can provide further insight into dynamics of processes on consumer markets where the social media play crucial role in spreading of information.
\end{abstract}

Keywords: Just Noticeable Difference, Marketing, Simulation, Agent-based Modelling, Social Media. 


\section{Introduction}

The concept of Just Noticeable Difference (JND) is widely used in the fields of physiology, psychology of perception, consumer behaviour and marketing practice. JND generally refers to a relative threshold in perception by humans. When a change in stimulus value (i.e. change of perceived characteristic) reaches the threshold, the change becomes recognized. When the stimulus value change is below the threshold, the change is not being recognized.

The problem with the concept of JND tactics use in marketing is that it is historically grounded in individual psychology and thus it doesn't respect social relationships and recent widespread use of social media. It is easily possible to imagine a situation (and it has happened in reality) when such a small change, although below average JND, would be recognized by only a few customers who would share their discovery online. If these customers would be able to start an information avalanche in social media then it could lead to a huge feeling of deception amongst all the other customers and a loss of trust.

For these reasons it is needed to rethink the concept of JND and validate whether and how these changed circumstances would influence its' marketing use in online environments.

Because such experimentation would be very complicated in the real world, it is necessary to use another approach which would allow to create and explore such an online environment artificially. Several possibilities exist for that, i.e. system dynamics, mathematical modelling or agent based modelling. For this study the author has chosen the agent based modelling (ABM) because this approach is especially suitable for problems where heterogeneous agents and their local interactions are sources of the overall emerging dynamics and it is possible to describe behaviour of agents using comprehensible algorithms based on existing knowledge.

For this case, the agents represent consumers and links between them represent social relationships. Then it's possible to define rules of behaviour of such agents and simulate and analyse diffusion processes that take place in such an artificial social network.

\section{Literature Review and Research Methods}

\subsection{The Concept of Just Noticeable Difference in Marketing}

The original concept of Just Noticeable Difference was published by Ernst Heinrich Weber in 1834. The Weber's Law states that the ratio of the increment threshold to the background intensity is constant (see Equation 1).

$$
\frac{\Delta I}{I}=k
$$

$\Delta I$ represents the Just Noticeable Difference threshold, $I$ represents the initial stimulus value and $k$ is constant.

The first notion of the Just Noticeable Difference concept in marketing has been probably made by Miller (1962). Since that time, it has become a part of practically all main consumer behaviour textbooks, e.g. Solomon (2014), Schiffman \& Wisenblit (2014) or Evans, Foxall, \& Jamal (2009), and thus also marketing practices.

The abovementioned authors argue, that in the context of marketing, tactics based on changes below JND are typically recommended for such changes of products that should not be easily recognized by customers - e.g. product size changes without packaging size changes, changes in taste, graphical changes in webpages, logotypes or packaging, gradual rebranding, small price or provided services changes etc. This should allow marketers to make changes that 
would be otherwise negatively perceived by their customers or gradually change their customers' perception standards towards new preferred state.

On the other hand, it might be also necessary to do the opposite and inform customers about certain changes. Then the change of stimulus value has to be significant enough to exceed the JND and the main concern of marketers is then to be able to inform their customers as efficiently as possible about the change.

Although nowadays the concept of JND in its' narrow interpretation is typically a matter of research in sensory perception analysis, marketing studies related to JND in broader terms can also be found - i.e. for package downsizing (Çakır \& Balagtas, 2014), word-of-mouth marketing (King, Racherla, \& Bush, 2014), website changes (Ainsworth \& Ballantine, 2014) or price changes (Han, Gupta, \& Lehmann, 2001).

\subsection{Modelling Diffusion of Information in Online Social Media Using Agent Based Modelling (ABM)}

In general, a diffusion of information through online social networks can be modelled using formal models similar to those that are used in epidemiology for modelling of spreading of infection diseases (Anderson \& May, 1992). These models can be created either on macro (equations describing dynamics of whole population - e.g. system dynamics, econometric models) or on micro level (nodes, agents and their interactions).

The use of ABM in this context has according to Schramm, Trainor, Shanker, \& $\mathrm{Hu}$ (2010) and Rand \& Rust (2011) several advantages - agent behaviour can be grounded in proper theories and influenced by other agents as well as by global and local settings (e.g. demographic attributes). This allows to respect heterogeneity when needed and to see emergent result on global scale.

The ABM has been extensively used for modelling of diffusion processes in social networks (Rand \& Rust, 2011). For instance, Delre, Jager, \& Janssen (2007) and Peres (2014) studied diffusion dynamics of new products according to different social network topologies. Watts \& Dodds (2007) studied impact of influentials in diffusion. Goldenberg, Han, Lehmann, \& Hong (2009) investigated network hubs influence. Schramm et al. (2010) focused their research on diffusion respecting brand influence, pricing, individual consumer characteristics and social influence and Kvasnička (2014) studied viral video diffusion in a fixed network.

To be able to draw meaningful conclusions, agent based models of diffusion process in online social networks have to be based on network topologies that properly reflect structure of the real online social networks.

The evolution of approaches to model such a network using computers has gone through several influential milestones. Watts \& Strogatz (1998) have introduced small world effect emergence which mimics one important feature of social networks - that nodes in such networks are both well connected and with large clustering coefficients. Barabási \& Albert (1999) proceeded further with a principle of preferential attachment building of social networks which allows to model scale-free networks. Holme \& Kim (2002) proposed a way how to complement preferential attachment models with triad formation which generates networks with both scale-free and small world properties. Pasta, Zaidi, \& Rozenblat (2014) added principles how to involve demographic properties to such networks. And Li et al. (2014) showed how it is possible to artificially build social networks which are sparse/dense and assortative/dissassortative and where these properties my change over the social network evolution. 


\section{Solutions and Results}

\section{1 $\quad$ Agent Based Model}

To validate the marketing tactics based on JND an information diffusion agent based model has been developed using Netlogo (Wilensky, 1999). Agents in this model represent consumers which have undirected links to others which shape their social interactions - in this case information spreading and receiving.

The model is based on network topology building algorithm suggested by Li et al. (2013) and Li et al. (2014) which allows to generate artificial social networks with realistic properties, a given average degree (amount of social relationships) and amount of nodes (agents, i.e. consumers).

The artificial social network of 1500 nodes used in this study was created using the following algorithm:

1. At the beginning, a network of few nodes is created with random links.

2. At every following step, a new node is created and randomly connected to one of existing nodes and a certain amount of activated nodes is randomly selected to connect to one of their unconnected second neighbour nodes. If there are no activated nodes available, nodes for connection are being chosen fully on random basis.

The nodes in the network are being activated when their state function $\varphi(i, t)$ exceeds a given threshold ( $i$ denotes the node index, $t$ denotes the time step). The state function is calculated using the following reaction-diffusion-like equation (Li et al., 2013):

$$
\varphi(i, t+1)-\varphi(i, t)=\varphi_{0}+\mu \sum_{j}^{N} S_{i j}\left[k_{j}(t+1)-k_{j}(t)\right]
$$

where $\varphi_{0}$ and $\mu$ are constants and summation represents changes in degrees of all neighbouring nodes $k_{j}$ ( $j$ denotes the neighbour node index). During the time, the state function value is increasing for each node until it reaches threshold value. Then the node is available for new connections and the function value is reset to zero in the next time step. Another situation when the value is being reset to zero is when a new connection with this node is being made - either randomly or based on actions of the other nodes.

Because the main purpose of this agent based model is to validate JND marketing tactics it was necessary to incorporate the concept of JND into the model. It has been done by adding variable of JND-threshold to each agent. The value is randomly generated when agent is created and in this case follows normal distribution with the mean $=0.5$ and standard deviation $=0.1$ (meaning that $96 \%$ of individual JND thresholds will be between 0.3 and $0.7)$.

It can be assumed that the agents can get information about given change either through their own experience with the product (when the change is above their individual JND-threshold) or through social media - in this case the value of their individual $J N D$-threshold is irrelevant. To reflect different situations that may occur, global variables size-of-change, amount-ofinfluenced-per-tick, probability-of-information-spreading and probability-of-informationreceiving have been added to the model.

The variable size-of-change denotes the relative change that is being done by marketers. It is quantified on a scale between 0 and 1 and the value can be easily compared to the individual $J N D$-threshold of each agent. Using this comparison it is possible to investigate effects of changes below and above the average JND. 
The variable amount-of-influenced-per-tick represents an amount of randomly selected agents that are directly influenced by the abovementioned change in each time step. It is possible to imagine them as customers buying or consuming the products in the given time. The value has been set up to 20 for all the following scenarios - so we can expect each agent to be selected on average 4 times during the simulation.

The variables probability-of-information-spreading and probability-of-information-receiving denote agents' propensity to spread the information to their peers and probability of receiving the information through social network.

The algorithm of information spreading is being implemented in the following very simple sequence for each time step (tick):

1. All the red agents send with the given probability-of-information-spreading a message to their social neighbours and turn brown.

2. Receiving agents with the given probability-of-information-receiving turn red.

3. Amount-of-influenced-per-tick agents are randomly selected and their individual JNDthreshold is being compared with the induced size-of-change - if the threshold is below the change size and it is the first time for the agent to get such an information, agents will change their colour to red.

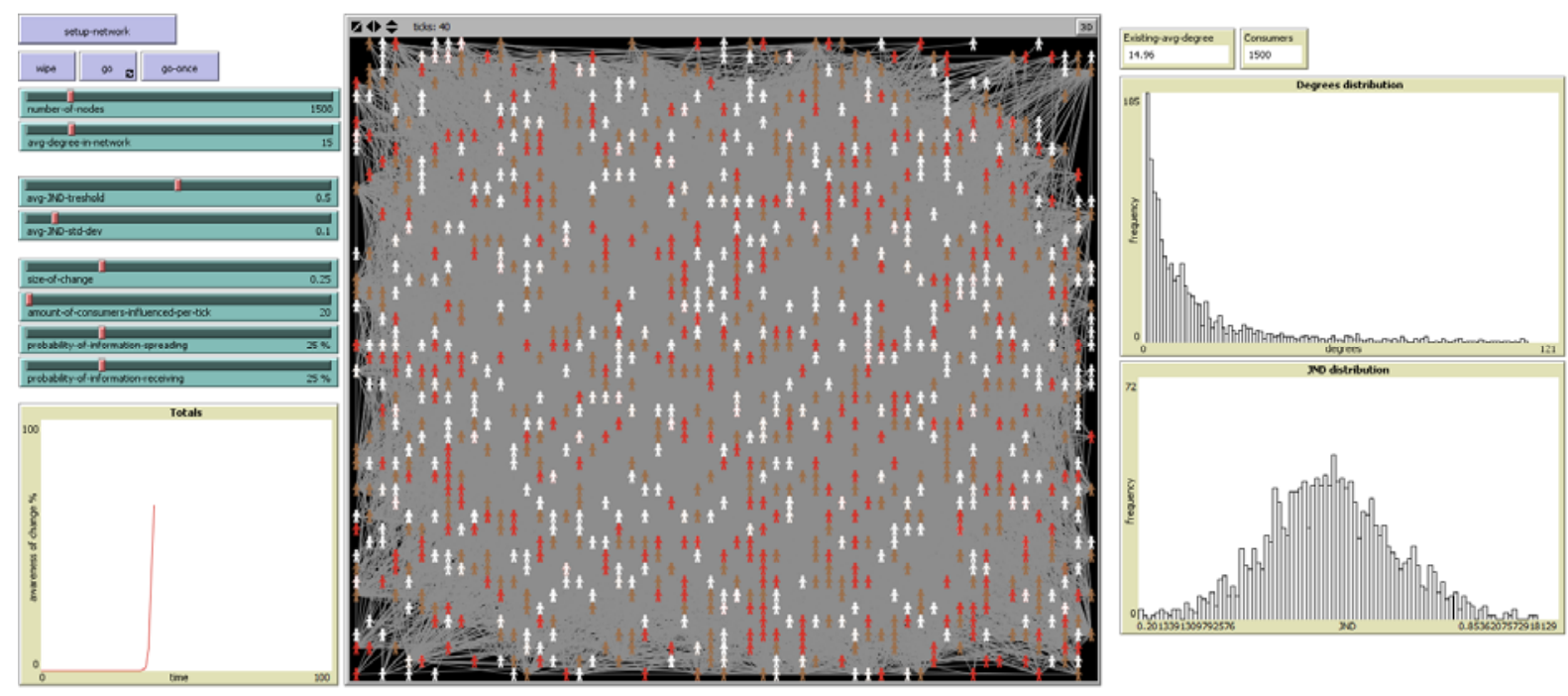

Fig. 1. User interface of the created agent based model. Source own elaboration.

\subsection{Simulated Scenarios}

For the validation of JND marketing tactics with the agent based model, 2700 runs have been simulated using BehaviorSpace function of NetLogo and the process described in Fig. 2. 


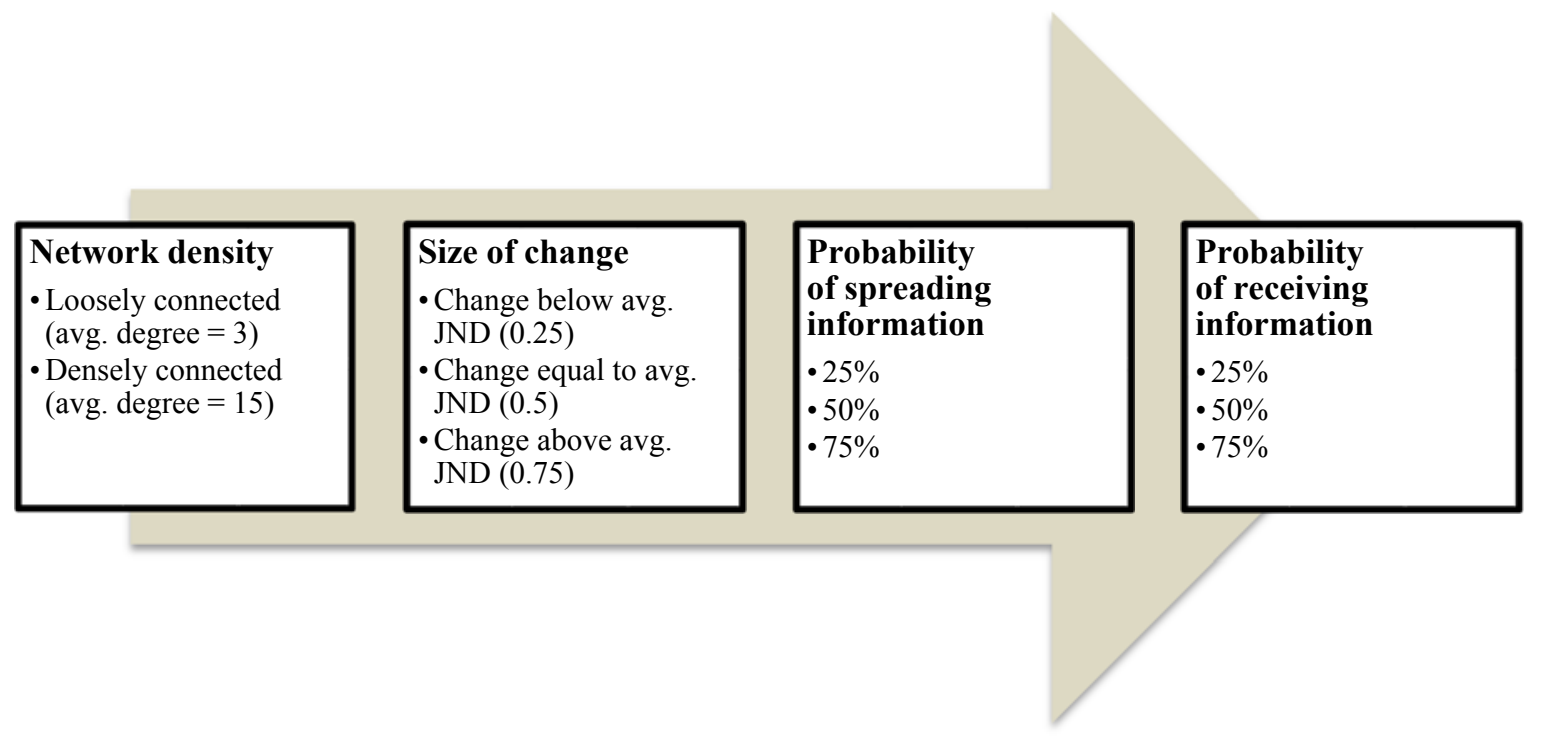

Fig. 2. Simulated scenarios. Source own elaboration.

At first, a loosely connected social network with average degree 3 has been generated. This setup reflects the situation before social media have been introduced. Then each of 27 following combinations (size of change vs. probability of spreading information vs. probability of receiving information) was simulated 50 times and data about diffusion of information were analysed as follows in the Section 3.3.

As the next step, a densely connected social network with average degree 15 has been generated which reflects online social media and easiness of information sharing. Then again each of 27 combinations was simulated 50 times and data about diffusion of information were analysed as follows in the Section 3.4.

\subsection{Loosely Connected Social Network Simulation Results}

It is possible to see the results for simulations in the loosely connected social network in Figures $3-5$. Figure 3 shows diffusion of information for changes below the average JND threshold, Figure 4 shows the same for changes equal to the average JND threshold and Figure 5 shows results for changes above the average JND threshold.

The results for small changes below the average JND threshold generally show that under these circumstances there could be a lot of variance in results and when probabilities of spreading and receiving information are low, it is possible on average to achieve very low and close to zero coverage of population. It might suggest that practices based on changes below JND might really work very well and the risk for marketers if the change is not favourable to customers is very low too. This might explain why JND marketing tactics used to be working well in practice in the past. 


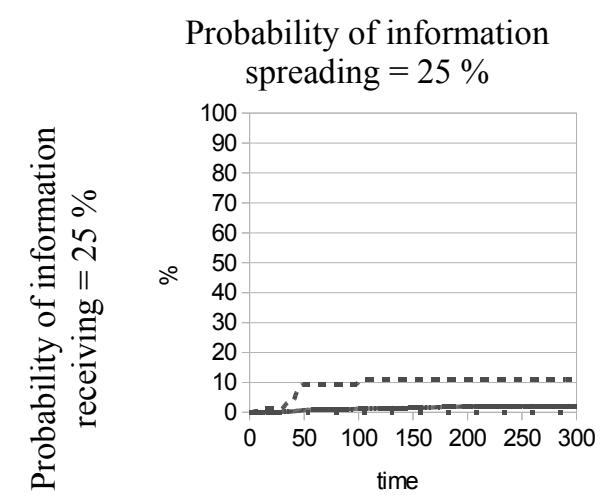

Probability of information spreading $=50 \%$
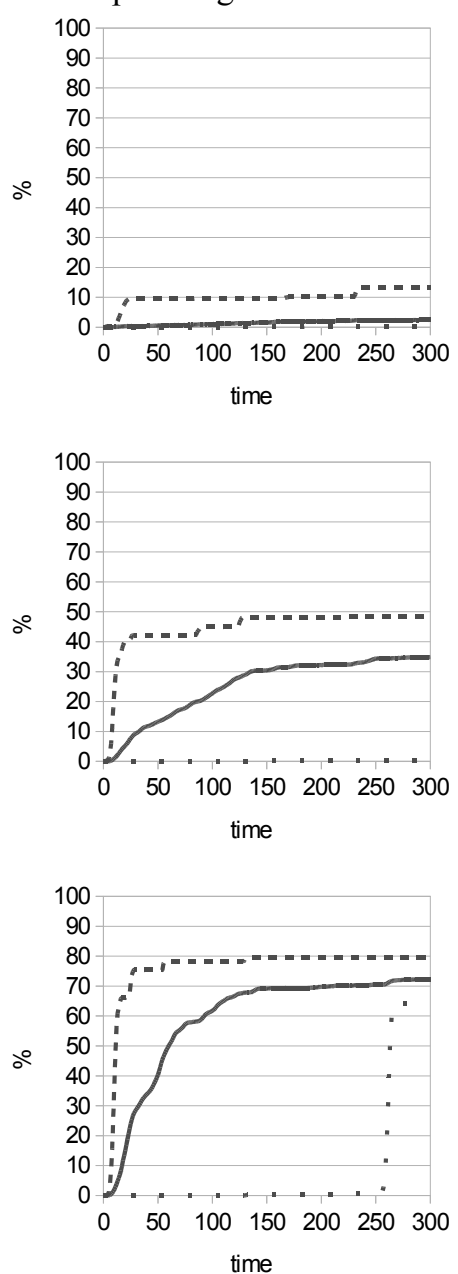

Probability of information spreading $=75 \%$
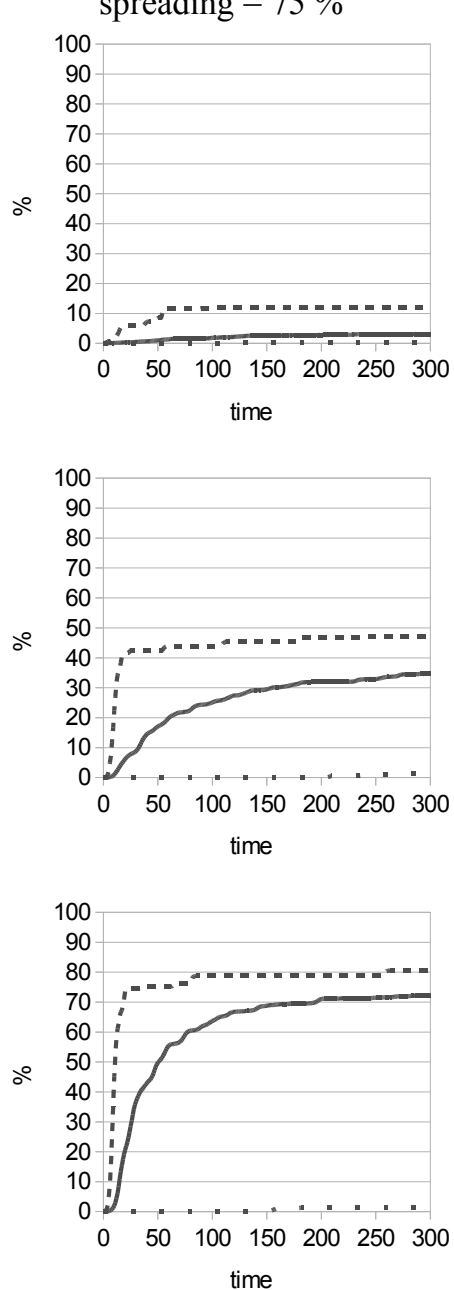

Nodes $=1500$, average degree in network $=3$, average JND treshold $=0.5$, average JND standard deviation $=0.1$, size of change $=0.25$, amount of consumers influenced per tick $=20$

Solid lines are average values, dashed lines are maximum values, dotted lines are minimum values for 50 simulation runs

Fig. 3. Diffusion of information for changes below average JND threshold, loosely connected social network.

Source own elaboration. 
When the changes are equal to the average JND threshold, variance in the diffusion becomes very low and it is possible to see slightly different diffusion patterns according to the different probabilities of spreading and receiving information. With higher probabilities, the information diffusion is generally faster and covers higher proportion of population.
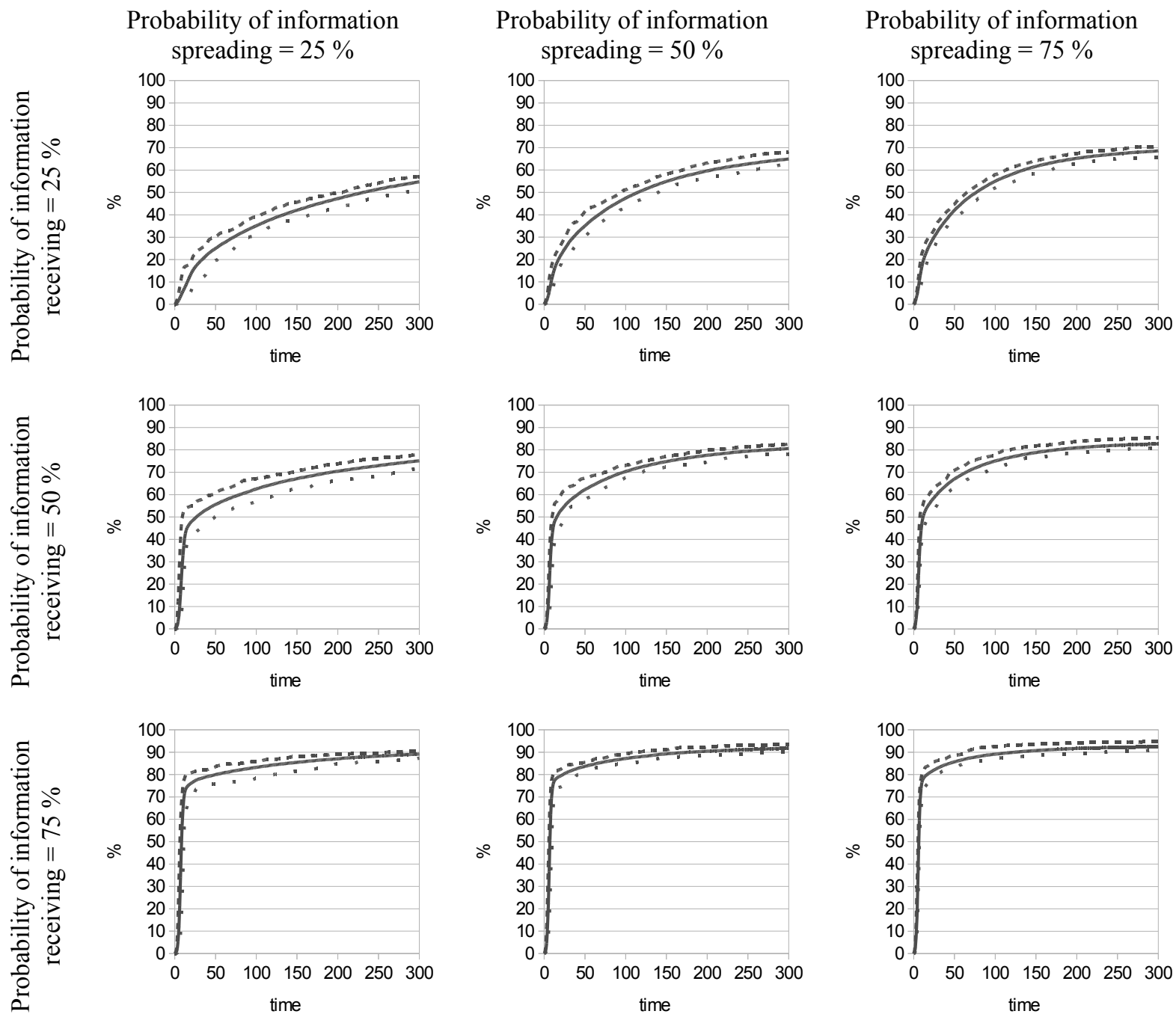

Nodes $=1500$, average degree in network $=3$, average JND treshold $=0.5$, average JND standard deviation $=0.1$, size of change $=0.50$, amount of consumers influenced per tick $=20$

Solid lines are average values, dashed lines are maximum values, dotted lines are minimum values for 50 simulation runs

Fig. 4. Diffusion of information for changes equal to average JND threshold, loosely connected social network. Source own elaboration. 
The results for changes above the average JND threshold are very similar as the previous ones, the only difference is in higher speed and proportion of population being covered by the information diffusion.
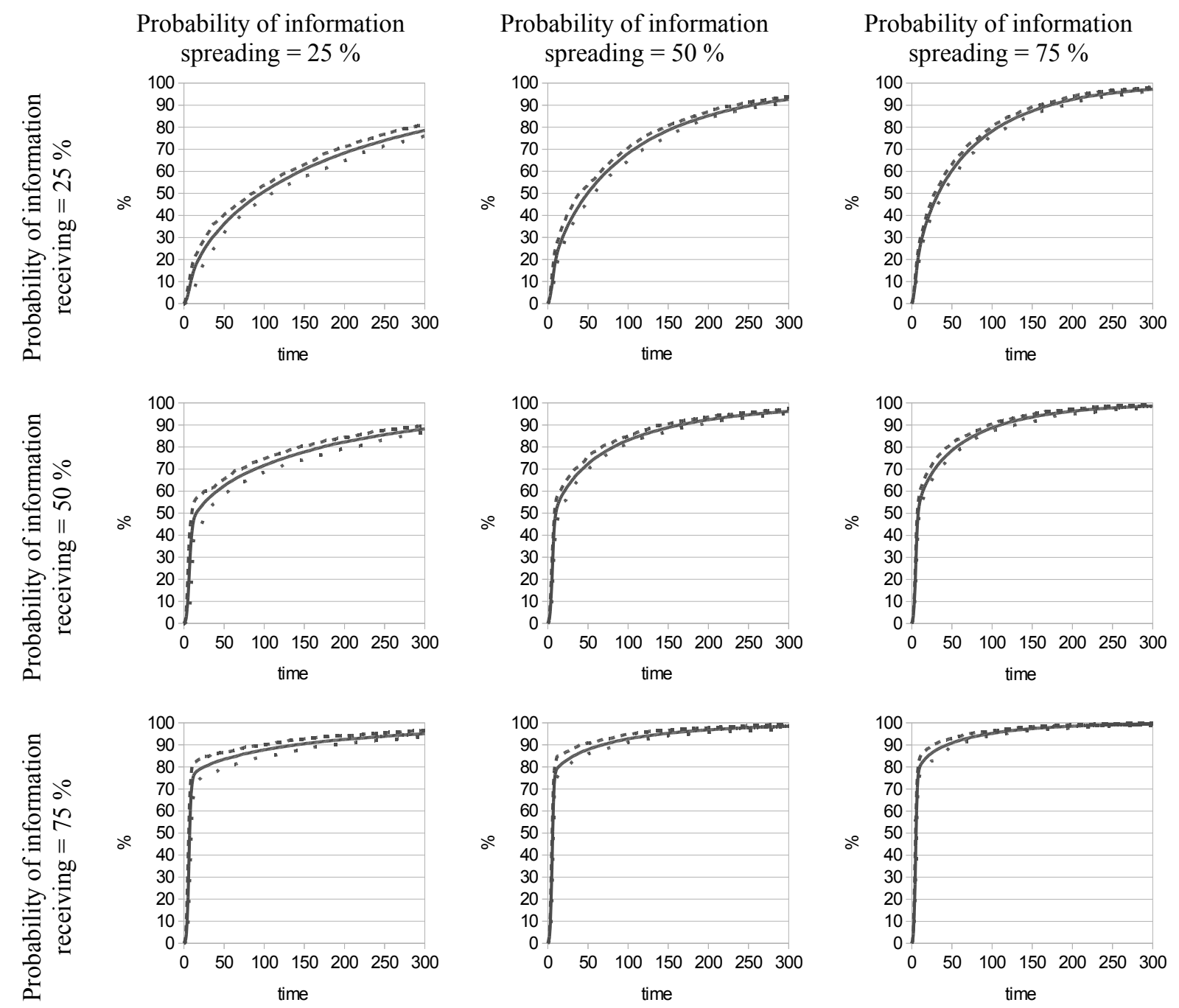

Nodes $=1500$, average degree in network $=3$, average JND treshold $=0.5$, average JND standard deviation $=0.1$, size of change $=0.75$, amount of consumers influenced per tick $=20$

Solid lines are average values, dashed lines are maximum values, dotted lines are minimum values for 50 simulation runs

Fig. 5. Diffusion of information for changes above average JND threshold, loosely connected social network. Source own elaboration.

\subsection{Densely Connected Social Network Simulation Results}

Again, it is possible to see the results for simulations in the densely connected social network in Figures $6-8$. Figure 6 shows the diffusion of information for changes below the average JND threshold, Figure 7 shows the same for changes equal to the average JND threshold and Figure 8 shows results for changes above the average JND threshold.

In comparison to the results for the loosely connected social network it is possible to see a dramatic change in the increase of population information coverage even when the probabilities of spreading and receiving information are low and the change is below the JND threshold. 
The importance of such diffusion pattern change is clearly visible not just on average but also on minimum values (dotted lines) in Fig. 6.

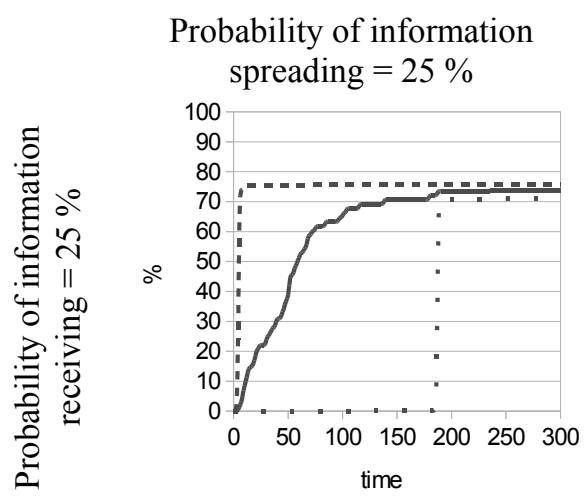

\section{Probability of information spreading $=50 \%$}

time

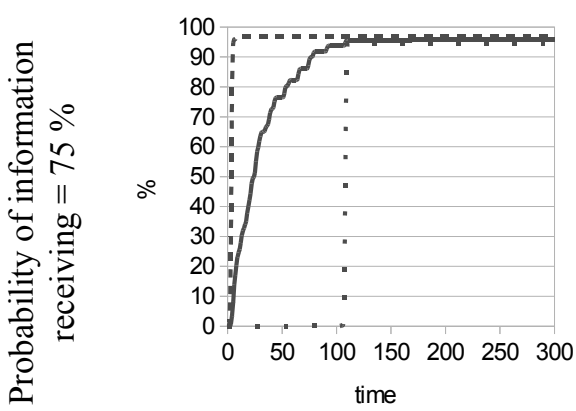

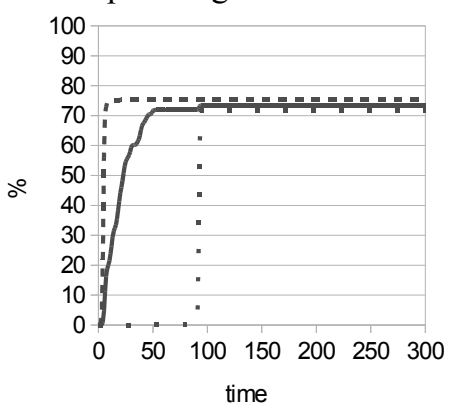

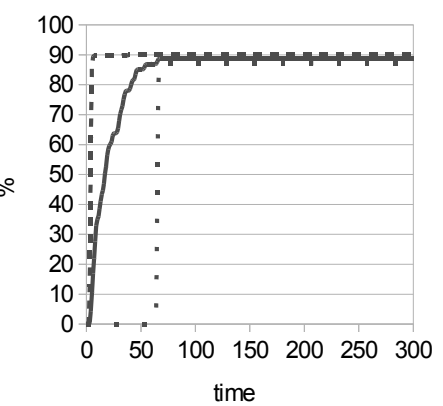

Probability of information spreading $=75 \%$
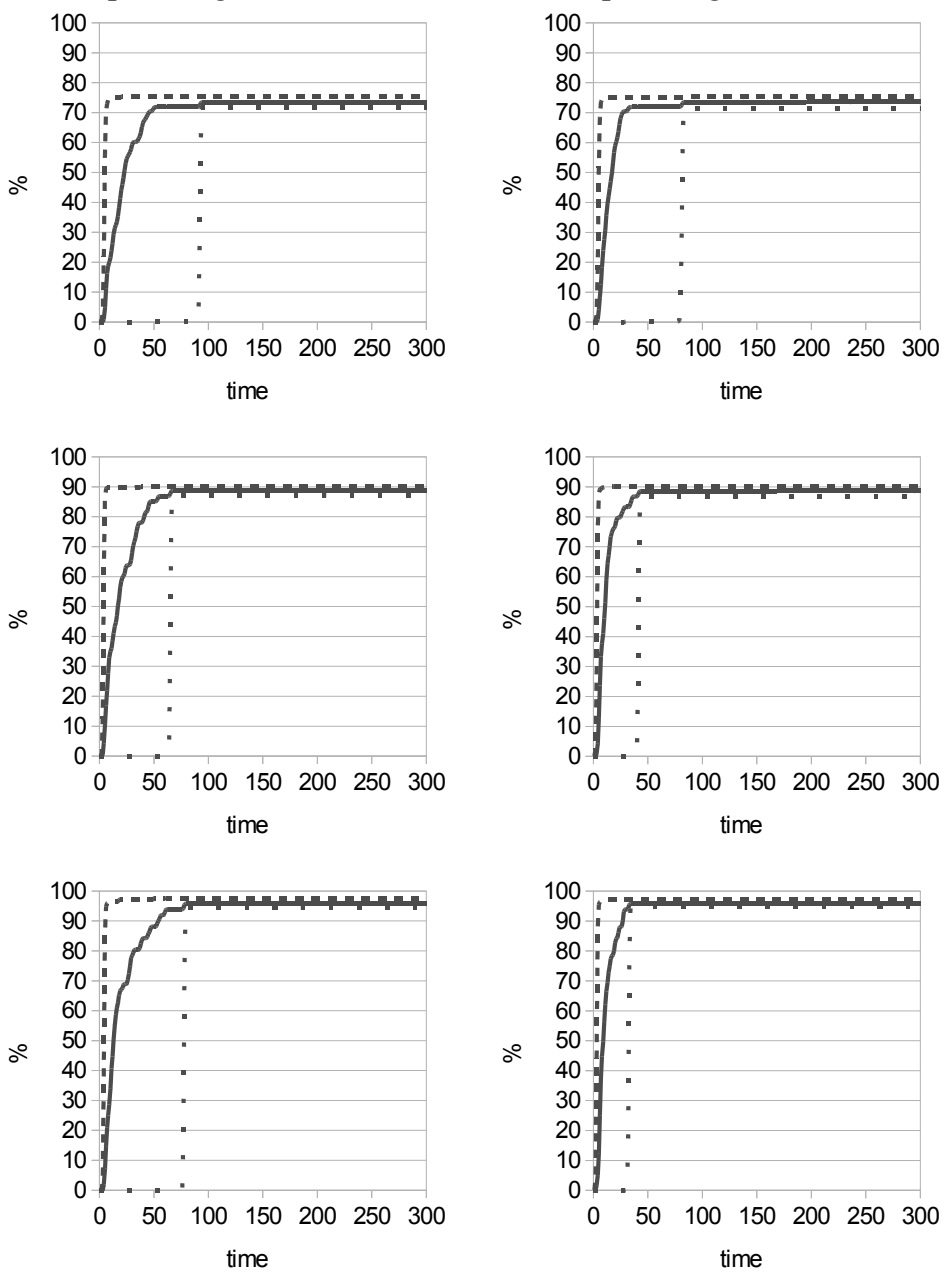

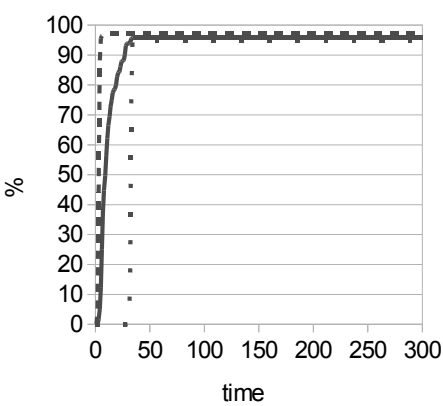

Nodes $=1500$, average degree in network $=15$, average JND treshold $=0.5$, average JND standard deviation $=0.1$, size of change $=0.25$, amount of consumers influenced per tick $=20$

Solid lines are average values, dashed lines are maximum values, dotted lines are minimum values for 50 simulation runs

Fig. 6. Diffusion of information for changes below average JND threshold, densely connected social network. Source own elaboration. 
When the results for changes equal to the average JND threshold are being compared to the loosely connected social network, the coverage of population is faster and higher.

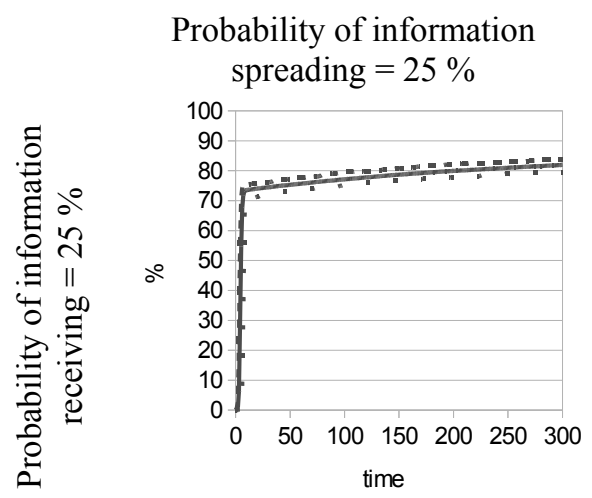

\section{Probability of information spreading $=50 \%$}

time

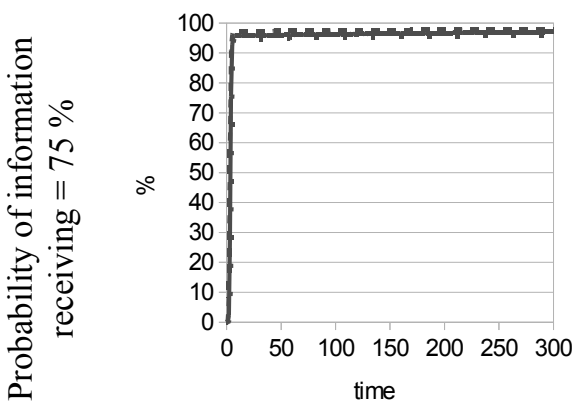

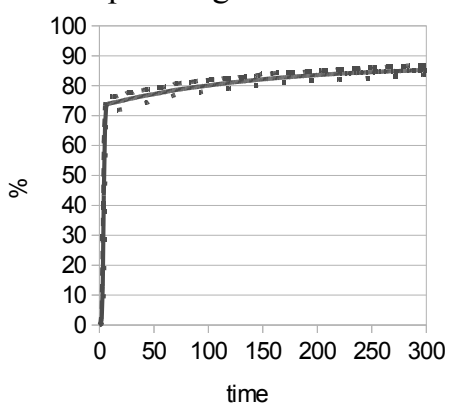

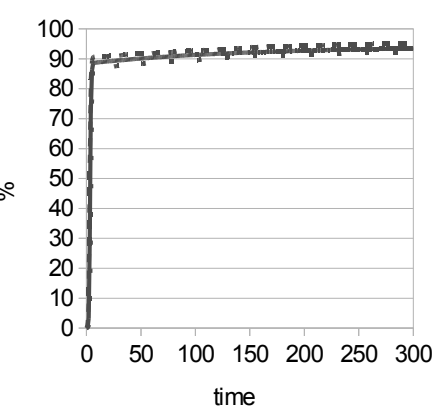

Probability of information spreading $=75 \%$
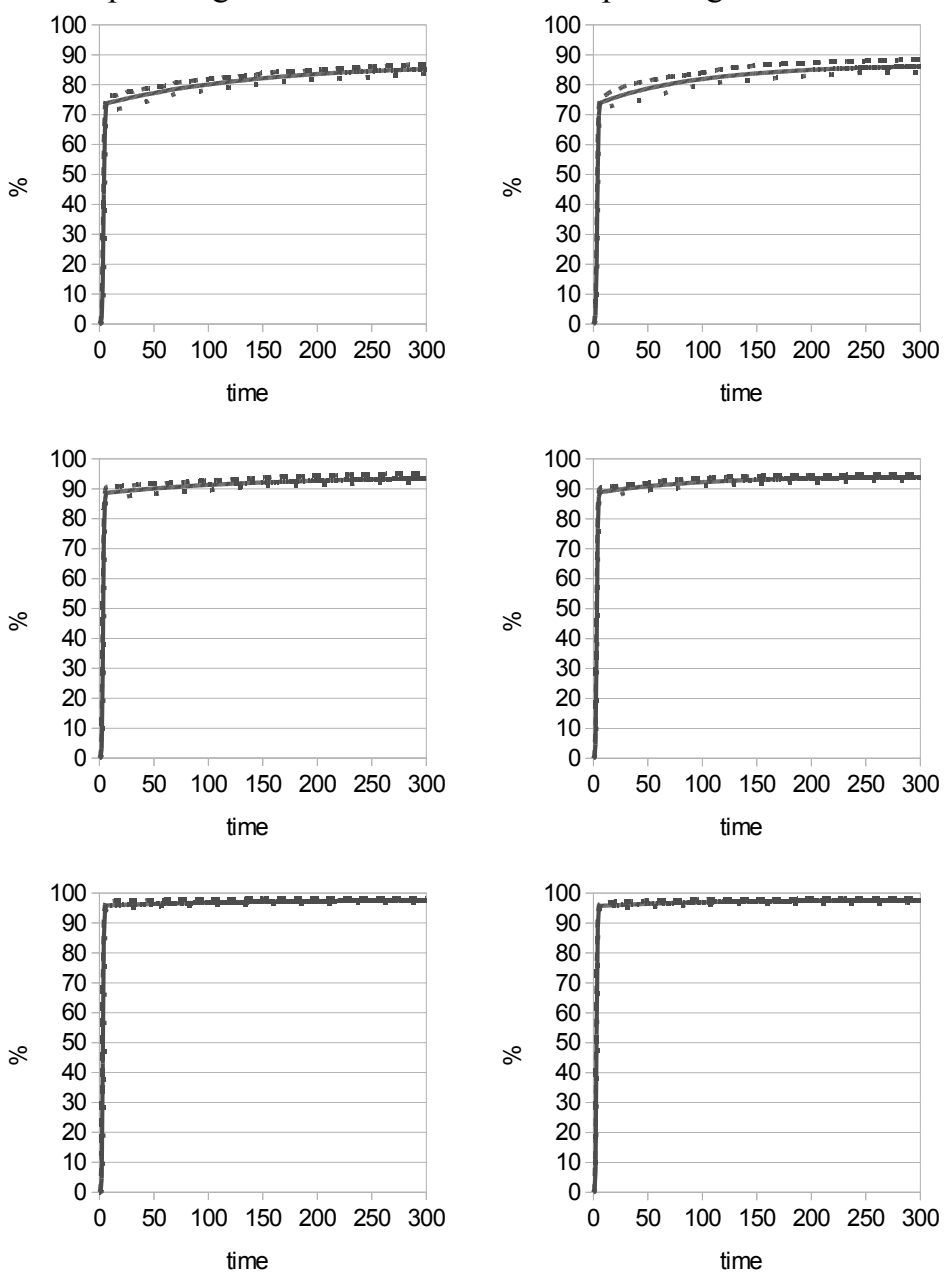

Nodes $=1500$, average degree in network $=15$, average JND treshold $=0.5$, average JND standard deviation $=0.1$, size of change $=0.50$, amount of consumers influenced per tick $=20$

Solid lines are average values, dashed lines are maximum values, dotted lines are minimum values for 50 simulation runs

Fig. 7. Diffusion of information for changes equal to average JND threshold, densely connected social network. Source own elaboration. 
The results for changes above the average JND threshold are very similar as the previous ones, the only difference is again in higher speed and proportion of population being covered by the information diffusion.
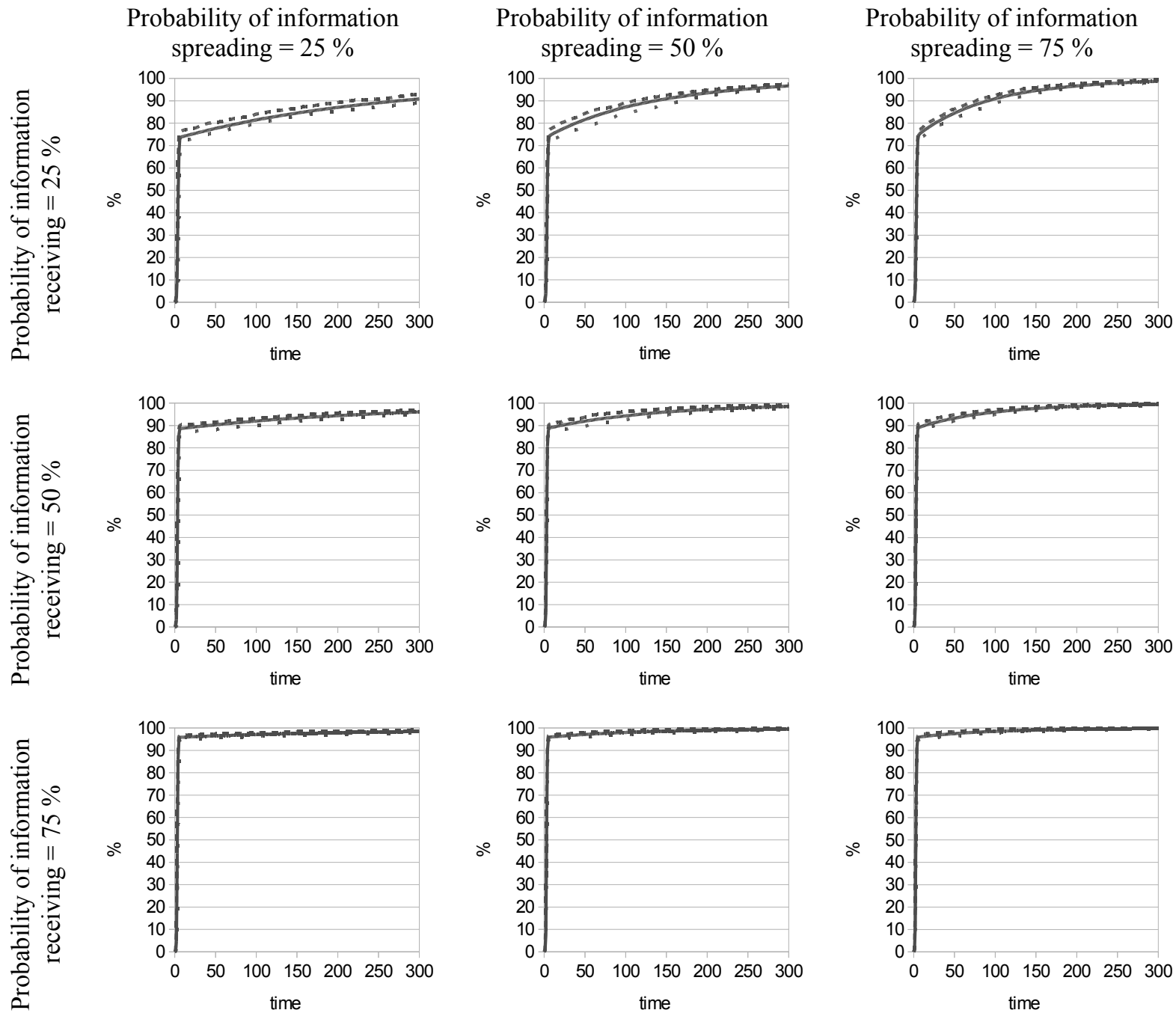

Nodes $=1500$, average degree in network $=15$, average JND treshold $=0.5$, average JND standard deviation $=0.1$, size of change $=0.75$, amount of consumers influenced per tick $=20$

Solid lines are average values, dashed lines are maximum values, dotted lines are minimum values for 50 simulation runs

Fig. 8. Diffusion of information for changes above average JND threshold, densely connected social network. Source own elaboration.

\section{Discussion and Conclusion}

When comparing the overall results, it is clear that higher average degree of the simulated social network lead to faster diffusions which also covered higher proportions of population. If the changes below the average JND are being analysed in more detail, the results suggest that in the world without social media (i.e. with low average degrees in the network of social relationships) it would be possible to easily "hide" the changes below the average JND threshold. 
On the other hand, in the world with social media (i.e. with higher degrees in the network of social relationships) the results are dramatically different and in all simulations at least $70 \%$ of population got the information later or sooner. This means that also the level of risk for marketers using JND tactics should increase significantly in such circumstances and these practices could be harmful for companies. This is also at least partially in contrary to the older consumer behaviour and marketing findings and recommendations as mentioned in the literary review which do not take these influences into consideration.

In all the scenarios of changes below the average JND threshold less than $1 \%$ of consumers were able to recognize the change, meaning that only their individual JND thresholds were above the size of change being applied by marketers. Even such a small proportion was able to start information avalanches through social networks as can be seen on Fig. 6 on maximum values for the proportion of population being covered by the information diffusion.

For the changes equal to the average JND threshold the information diffusion was only partially successful for the world without social media where the proportion of population was less than $60 \%$ after 300 time steps of simulation. On the other hand, in this case it was just a matter of time to get to higher population proportions. From marketing perspective, it would be meaningful in such situation to use other than social media to support information diffusion.

In the world with social media the information spreads much faster and all the simulations got to $70 \%$ of population in a very short time even though each node sends information just once. In this situation it would be meaningful for marketers to support not the other media but diffusion processes between consumers which could be more efficient due to lower costs. The very similar suggestion applies to changes bigger than the average JND threshold.

The study presented here has of course certain limitations. One of the limitations is that only two artificially generated social networks were used. On the other hand the algorithm for their building was properly tested and validated in previous studies (Li et al., 2013) and further tests with different social networks generated by the same algorithm showed consistent results. And because the findings are more of qualitative nature this limitation should not undermine them.

Another limitation could be related to the size of social network being simulated. Because the building of such a social network is dependent on a computer processing power and time available, size of 1500 nodes was selected for experiments as a compromise. Bigger networks (10000 nodes) were tested too to see whether the results are consistent and the behaviour patterns were fully comparable.

This study had one main goal - to answer the question whether the just noticeable difference related marketing practices could survive in the world with social media and as a part of online marketing.

Although the findings are limited, they suggest that using of such practices might be much riskier than it used to be before and marketers should be aware of that and consider their using more thoroughly. In this sense, also consumer behaviour and marketing textbooks should be updated accordingly.

It was also shown that the agent based modelling can be helpful in dealing with problems like this one and can provide further insight into dynamics of processes on consumer markets where the social media play crucial role in spreading of information.

\section{Acknowledgement}

This paper has been supported from a project GAJU 79/2013/S - Rolínek. 


\section{References}

Ainsworth, J., \& Ballantine, P. W. (2014). That's different! How consumers respond to retail website change. Journal of Retailing and Consumer Services, 21(5), 764-772. doi:10.1016/j.jretconser.2014.06.003

Anderson, R. M., \& May, R. M. (1992). Infectious Diseases of Humans: Dynamics and Control (Reprint edition.). Oxford University Press.

Barabási, A.-L., \& Albert, R. (1999). Emergence of scaling in random networks. Science, 286(5439), 509-512. doi:10.1126/science.286.5439.509

Çakır, M., \& Balagtas, J. V. (2014). Consumer Response to Package Downsizing: Evidence from the Chicago Ice Cream Market. Journal of Retailing, 90(1), 1-12. doi:10.1016/j.jretai.2013.06.002

Delre, S. A., Jager, W., \& Janssen, M. A. (2007). Diffusion dynamics in small-world networks with heterogeneous consumers. Computational and Mathematical Organization Theory, 13(2), 185-202. doi:10.1007/s10588-006-9007-2

Evans, M. M., Foxall, G., \& Jamal, A. (2009). Consumer Behaviour (2nd edition.). Chichester, England; Hoboken, NJ: Wiley.

Goldenberg, J., Han, S., Lehmann, D. R., \& Hong, J. W. (2009). The Role of Hubs in the Adoption Process. Journal of Marketing, 73(2), 1-13. doi:10.1509/jmkg.73.2.1

Han, S., Gupta, S., \& Lehmann, D. R. (2001). Consumer price sensitivity and price thresholds. Journal of Retailing, 77(4), 435-456. doi:10.1016/S0022-4359(01)00057-4

Holme, P., \& Kim, B. J. (2002). Growing scale-free networks with tunable clustering. Physical Review E Statistical, Nonlinear, and Soft Matter Physics, 65(2), 026107/1-026107/4. doi:10.1103/PhysRevE.65.026107

King, R. A., Racherla, P., \& Bush, V. D. (2014). What We Know and Don't Know About Online Word-ofMouth: A Review and Synthesis of the Literature. Journal of Interactive Marketing, 28(3), 167-183. doi:10.1016/j.intmar.2014.02.001

Kvasnička, M. (2014). Viral Video Diffusion in a Fixed Social Network: An Agent-based Model. Procedia Economics and Finance, 12, 334-342.

Li, M., Guan, S., Wu, C., Gong, X., Li, K., Wu, J., ... Lai, C.-H. (2014). From sparse to dense and from assortative to disassortative in online social networks. Scientific Reports, 4. doi:10.1038/srep04861

Li, M., Zou, H., Guan, S., Gong, X., Li, K., Di, Z., \& Lai, C.-H. (2013). A coevolving model based on preferential triadic closure for social media networks. Scientific Reports, 3. doi:10.1038/srep02512

Miller, R. L. (1962). Dr. Weber and the Consumer. Journal of Marketing, 26(1), 57-61. doi:10.2307/1249633

Pasta, M. Q., Zaidi, F., \& Rozenblat, C. (2014). Generating online social networks based on socio-demographic attributes. Journal of Complex Networks, cnu034. doi:10.1093/comnet/cnu034

Peres, R. (2014). The impact of network characteristics on the diffusion of innovations. Physica A: Statistical Mechanics and Its Applications, 402, 330-343. doi:10.1016/j.physa.2014.02.003

Rand, W., \& Rust, R. T. (2011). Agent-based modeling in marketing: Guidelines for rigor. International Journal of Research in Marketing, 28(3), 181-193. doi:10.1016/j.ijresmar.2011.04.002

Schiffman, L. G., \& Wisenblit, J. (2014). Consumer Behavior (11 edition.). Boston: Prentice Hall.

Schramm, M. E., Trainor, K. J., Shanker, M., \& Hu, M. Y. (2010). An agent-based diffusion model with consumer and brand agents. Decision Support Systems, 50(1), 234-242. doi:10.1016/j.dss.2010.08.004

Solomon, M. R. (2014). Consumer Behavior: Buying, Having, and Being (11 edition.). Boston: Prentice Hall.

Watts, D. J., \& Dodds, P. S. (2007). Influentials, Networks, and Public Opinion Formation. Journal of Consumer Research, 34(4), 441-458. doi:10.1086/518527

Watts, D. J., \& Strogatz, S. H. (1998). Collective dynamics of "small-world” networks. Nature, 393(6684), 440442. doi:10.1038/30918 
Wilensky, U. (1999). NetLogo. Evanston, IL: Center for Connected Learning and Computer-Based Modeling, Northwestern University. Retrieved from http://ccl.northwestern.edu/netlogo/ 